\title{
EXAMINATION OF THE EFFECT OF PSYCHOPHYSICAL FACTORS ON THE QUALITY OF HUMAN GAIT RECOGNITION
}

\author{
Marcin DERLATKA* \\ * Department of Automatic Control and Robotics, Faculty of Mechanical Engineering, Bialystok University of Technology, \\ ul. Wiejska 45c, 15-351 Bialystok, Poland \\ m.derlatka@pb.edu.pl
}

\begin{abstract}
The paper presents an analysis concerning the influence of selected psychophysical parameters on the quality of human gait recognition. The following factors have been taken into account: body height $(\mathrm{BH})$, body weight (BW), the emotional condition of the respondent, the physical condition of the respondent, previous injuries or dysfunctions of the locomotive system. The study was based on data measuring the ground reaction forces (GRF) among 179 participants (3 315 gait cycles). Based on the classification, some kind of confusion matrix were established. On the basis of the data included in the matrix, it was concluded that the wrong classification was most affected by the similar weight of two confused people. It was also noted, that people of the same gender and similar BH were confused most often. On the other hand, previous body injuries and dysfunctions of the motor system were the factors facilitating the recognition of people. The results obtained will allow for the design of more accurate biometric systems in the future.
\end{abstract}

Key words: Human Gait Recognition, Ground Reaction Forces, Classification

\section{INTRODUCTION}

Among behavioral biometrics human gait deserves a particular attention. In comparison with other methods of person recognition gait measurement does not require any unnatural interaction of the person with the measuring devices and the person under examination does not have to be aware that is subject to this procedure. Increase in interest in gait as a biometric occurred in 2003, when the DARPA in the USA began its research on the project "Human Identification at a Distance" (Xu et al., 2013).

Currently, within the research on human gait biometrics, we can distinguish three main categories, depending on the signals registered. They are methods based on the data obtained from (Gafurov et al., 2011):

- cameras (Balista J. A. et al., 2010);

- instruments measuring interactions between the foot and the ground (Kumar and Ramakrishnan, 2011; Nakajima et al., 2000; Rodriguez et al., 2009; Yao et al., 2010);

- instruments, markers installed on the examined person (Klempous, 2012).

The most popular methods of human gait recognizing and simultaneously having the broadest spectrum of potential applications are based on measurements obtained from video cameras. In this case the picture registered is usually converted frame by frame into silhouette sequences. Subsequently, depending on the applied methods there occurs an attempt at reading of selected parameters of the human's gait and classification of a person. Advantages of these methods are undoubtedly a possibility of free motion of the person under examination, identification of many people simultaneously and these people's approval of the presence of cameras in buildings (Katiyar et al., 2013). Next group of methods based on the measurement of the interaction of the ground with the lower limbs of the person under examination. Here the examined person must walk along an appropriately-prepared measurement path equipped with force plates (Derlatka, 2012) or special foor with a network of photo interrupter sensors (Yun, 2011). In the case of these methods the main problem is an accurate hit in the measuring device.

In the last method mentioned above the person under examination fully cooperates during the time of measurement. He/she is provided with measurement equipment, such as: accelerators (Gafurov et al., 2011), opto-reflective markers (Lin et al., 2011), or else special insoles in the shoes registering foot pressures on the surface (Porwik et al., 2010).

The process of acquisition of the data describing a human's gait and potential extraction of its features is followed by a classification with such methods as neural networks (Lin and Lin, 2013), Naïve Bayes classifier (Switonski et al., 2011), genetic algorithms (Goldy and Mary, 2012) and others (Moustakidis et al., 2009).

It should be pointed out that very few research, analysing the impact of various parametres on the quality of the biometric system based on human gait, were published. Most often the subject area of acticles represents a choice of: measured signals (Lin and Lin, 2013) or method of features extraction (Pataky et al., 2012). One of the few exceptions, where factors imfluencing the quality of human gait recognition were mentioned, is (Katiyar et al., 2013) According to it problems with recognizing people in the systems based on video cameras are generated by the examined person's change of clothes, a possibility of covering the silhouette of the examined person by objects or other people, changes in lighting as well as the sensitivity of certain parameters sought for to the position angle of the object in relation to the camera

For the methods based on the measurement of the interaction between the foot and the ground practically only at work (Jenkins and Ellis, 2007), the influence of the weight of the participant on the obtained accuracy of the recognition has been tested. 
The authors used both normalised to the BW and non-normalised GRF as the input for the biometric system. The results for the nonnormalised GRF were significantlly weaker. It is also important to emphasize, however, that previous works were based on an exceptionally low number of people under examination (Gafurov et al., 2011; Lin et al., 2011; Moustakidis et al., 2009; Switonski al., 2011). Very few of scarce exceptions are (Derlatka, 2013; Pataky et al, 2012; Rodriguez et al., 2009), which presented a research based on the group of over 100 people. Unfortunately in those cases, the authors do not usually conduct an in-depth analysis of the results based on the presented material.

The main objective of this paper is to analyse of erroneous classifications in respect of selected psychophysical parameters like body weight, body height, emotions of subjects as well as injuries of organs of movement experienced in the past.

\section{METHODOLOGY}

\subsection{Materials}

179 people (66 women and 113 men) took part in the research conducted in the Bialystok University of Technology. It should be stressed out that it is the biggest database containing data describing human gait using GRF. The participants in the research were $21.28 \pm 1.04$ years old, body weight $75.49 \pm 16.64$ kilos and body height $174.95 \pm 9.29 \mathrm{~cm}$.

Before the research a questionnaire was carried out, where the people under examination provided information on their musculoskeletal dysfunctions and episodes from their past which may affect the gait pattern as well as about their current physicoemotional state.

On the basis of the questionnaires it was concluded that among the system users there were 70 people who did not suffer from any ailments of musculoskeletal system and did not report any incidents from the past. 43 volunteers in this group used to have sprained ankles, including 8 with both ankles. 28 people reported scoliosis, 9 unequal length of lower limbs with at least $0.5 \mathrm{~cm}$ of difference, 17 people had broken legs or briken foot's finger in the past, and 8 subjects have flat feet. Moreover, single reports referred to such injuries as: spinal disc herniation, chondromalacia patella, forefoot adduction, kyphosis, lordosis, foot without two fingers (I and II) etc.. In total, the participants reported 24 different types of injuries or dysfunctions of the musculoskeletal system.

Furthermore, in the questionnaire mentioned earlier, the participants determined emotions accompanying them at a given moment and described their physical condition. When determining emotions the participants could choose from the following: serenity, joy, anxiety, uncertainty, nervousness, rush, impatience, excitement, curiosity, irritability. As far as the physical condition is concerned, the participants could select one of the five following options: drowsy, slightly drowsy, well-rested, slightly tired, tired.

\subsection{Measurements}

This paper, as a basis for recognizing people by gait, adopted three component forces of the ground reaction force: vertial, anterior/posterior and medial/lateral. In the biomechanical sense the ground reaction force (GRF) is a force which affects the person under examination as a result of the ground reaction to pres- sure. GRF as well as COP are measured by means of the force plates. The detailed description of the force plates can be found in Idźkowski and Walendziuk (2009).

Peaks for the vertical component $F y$ correspond with the moments of: transferring the weight of the whole body on the limb measured (the first peak force: the peak forces during weight acceptance and the forefoot loading (heel off the ground) just before the toe-off moment; the second peak force: peak forces during terminal stance).

These peaks, for correct feet, reach approximately $120 \%$ of the body's weight. This results from the need for maintaining balance while walking. Therefore the value of the reaction forces is higher than the weight force. At the half of the stance period, the whole active surface of the foot touches the ground. This is a period of unloading force during mid-stance (a minimum of the unloading force).

The anterior/posterior component $F x$ has two phases. In the first one its value is negative. This is a result of decelerate of the registered lower limb: the direction of the force is opposite to the direction of walking. The minimum of the inhibiting phase is usually reached just before the peak force during the weight acceptance occurs for vertical component Fy. Analogically, in the second phase the anterior/posterior component takes positive values. Then there begins the process of acceleration completed with taking the toes off the ground. During the whole period the direction of force $F x$ is the same as the direction of walking. The peak of the acceleration phase occurs at the initial toe-off phase. This takes place the moment after maximum propulsion for vertical component $F y$. The value of component $F x$ equals zero at the moment of the rear leg is passing the front leg. This corresponds, more or less, with the moment of occurrence of the minimum unloading phase for vertical component $F y$. The extreme values of component $F x$ reach approximately $20 \%$ of the body weight of the person under examination.

The direction of the component $\mathrm{Fz}$ depends on the examined lower limb. Usually it is assumed that values of $F z$ are positive for the left lower limb and negative for the right lower limb. A slight exceptions are the moments of initial contact and toe-off, where the foot is at a slight supination. The value of force $F z$ depends on the style of putting feet on the ground by the person under examination. This force should be greater both in the case of foot pronation as well as in the case of foot abduction. Extremes for $F z$ are called the same as in the case of vertical component $F y$ : peak forces during weight acceptance and terminal stance, unloading-force during midstance. The values of these forces make approximately $10 \%$ of the examined person's body weight.

During the examinations the person moved at a free speed in his/her own sport shoes on the measurement path, in which 2 Kistler force plates were hidden, working at the frequency of $1 \mathrm{kHz}$. The volunteers performed several walks (14-20), as a result of which over 3300 strides were registered.

\subsection{Dynamic Time Warping}

The distance (similarity) between time series has been calculated based on well-known Dynamic Time Warping (DTW) algorithm (generalized the Levenshtein's distance). The result of DTW algorithm is the minimal cost of the so called warping paths, which adjust one time series to another. This investigation take into consideration all three components of two GRFs so the total distance The distance between $n$ and $m$ patterns has been calcu- 
lated according to following formula:

$D(n, m)=\sum_{c=1}^{6} D_{c}$

where: $D_{c}$ - is the DTW distance between the $c$-th component of GRF for patterns $n$ and $m$.

\subsection{Human gait recognition}

The recorded strides have been divided into two sets. 1432 patterns were treated as prototype points (a learning set) and 1883 patterns obtained were treated as a testing set.

The modified version of the $k-\mathrm{NN}$ algorithm, presented in Derlatka (2013), has been used for the data classification in this article. The author is aware that there are more advanced algorithms which enable to obtain better classification results. In this case, nonetheless, getting the best human recognition is not as important as the analysis of crucial factors that influence the quality of the recognition. It is only possible when one has a sufficient number of classifier errors which in turn are not random errors but exhibit a steady tendency. According to the author, this criterion is met by the modified $k-\mathrm{NN}$ version with a selected $\vartheta=0.4$ threshold.

The modified $k-N N$ classification algorithm was as follows (Derlatka M., 2013):

1. Determine distance $D$ of the biometric pattern under examination from all prototype patterns in the data base.

2. Select k prototype patterns whose distances $D$ to the pattern under examination are the shortest.

3. By majority vote determine the $I D$ of the user in the data base. If two or more users are equally numerously representted among the patterns selected in point 2 (or the remaining after rejection in point 4) - select the one whose average distance from the selected pattern is the shortest.

4. Reject k' prototypes, for which distance $D$ is longer than $\rho_{\vartheta i}$ for the given threshold $\vartheta$ of the user whose $I D$ was selected in point 3 .

5. Check in compliance with the procedure in point 3 , whether $I D$ for $K=k-k^{\prime}$ of prototypes remained unchanged. If so, we finish the classification assigning the examined biometric pattern to the $I D$ class. If not, return to point 3 .

6. In the case when $k=k^{\prime}(K=0)$, we recognize that a given biometric pattern cannot be classified in any of the classes at the assumed threshold $\vartheta$.

\subsection{Confusion matrix}

Lets $X=\left\{x_{1}, x_{2}, \ldots, x_{9}\right\}$, where: $x_{1}$ - user ID, $x_{2}-$ ID predicted by the biometrics system, $x_{3}$ - body heigh (BH), $x_{4}$ - body weight (BW), $x_{5}$ - emotional state, $x_{6}$ - physical state, $x_{7}$ - user's sex, $x_{8}$ - injures in the past $\{1-$ yes; $0-$ no $\}$, $x_{9}-$ classification $\{$ correct, bad\}.

The $x_{8}$ parameter does not distinguish between different types of past bodily injury or dysfunctions of the motor system which affect the movement pattern. Such a decision was made as the number of kinds of injuries is too big when compared with the number of incorrect classifications. As a consequence, it precludes conducting a reliable analysis.

Of course we have: $x_{1}=x_{2}=>x_{9}={ }^{\prime}$ correct $^{\prime}$

$x_{1} \neq x_{2}=>x_{9}={ }^{\prime} b a d^{\prime}$

Let's define sets $G$ and $B$ as:

$G=\left\{X: x_{9}=\right.$ 'correct $\left.^{\prime}\right\}$

$B=\left\{X: x_{9}={ }^{\prime} b a d^{\prime}\right\}$

where: $G \cup B=X$ and $G \cap B=\{\phi\}$

Next we created set $X^{\prime}=\left\{x_{1}^{\prime}, x_{2}^{\prime}, \ldots, x_{9}^{\prime}\right\}$ in the following way:

$M G=\left\{X^{\prime}: \bigwedge_{i, j=1, \ldots, n} G_{i} \times G_{j}\right\}$

where: $x_{1}^{\prime}=x_{1 i}, x_{2}^{\prime}=x_{1 j}, x_{9}^{\prime}=x_{9 i}, x_{3}^{\prime}=a b s\left(x_{3 i}-x_{3 j}\right)$, $x_{4}^{\prime}=\operatorname{abs}\left(x_{4 i}-x_{4 j}\right), x_{5}^{\prime}$ is equal 0 if $x_{5 i} \neq x_{5 j}$ and 1 otherwise, $x_{6}^{\prime}$ is equal 0 if $x_{6 i} \neq x_{6 j}$ and 1 otherwise, $x_{7}^{\prime}$ is equal 0 if $x_{7 i} \neq x_{7 j}$ and 1 otherwise, $x_{8}^{\prime}$ is equal 0 if $x_{8 i} \neq x_{8 j}$ and 1 otherwise

$M B=\left\{X^{\prime}: \bigwedge_{i=1, \ldots, m} B_{i}\right\}$

where: $\quad x_{1}^{\prime}=x_{1 i}, \quad x_{2}^{\prime}=x_{1 j}, \quad x_{9}^{\prime}=x_{9 i}, \quad x_{3}^{\prime}=a b s\left(x_{3 i}-\right.$ $\left.x_{3 \mid x_{2 i}}\right), x_{4}^{\prime}=\operatorname{abs}\left(x_{4 i}-x_{4 \mid x_{2 i}}\right), x_{5}^{\prime}$ is equal 0 if $x_{5 i} \neq x_{5 \mid x_{2 i}}$, and 1 otherwise, $x_{6}^{\prime}$ is equal 0 if $x_{6 i} \neq x_{6 \mid x_{2 i}}$ and 1 otherwise, $x_{7}^{\prime}$ is equal 0 if $x_{7 i} \neq x_{7 \mid x_{2 i}}$ and 1 otherwise, $x_{8}^{\prime}$ is equal 0 if $x_{8 i} \neq x_{8 \mid x_{2 i}}$ and 1 otherwise.

As a result we obtained confusion matrix $P$ as:

$P=M G \cup M B$

\section{RESULTS AND DISCCUSSION}

As a result of classifying participants based on the modified $k \mathrm{NN}$ algorithm, only 61 strides (3.21\%) were assigned to wrong person. This provides evidence showing high discriminatory abilities of human gait as biometrics and presented classification method.

According to (8) the matrix $\mathrm{P}$ has been created. It consists of 31868 patterns.

Figure 1 shows the percentage contribution of parametres such as: 'the same sex', 'the same emotions', 'the same physical condition', 'previous injuries', which amount equivalent to 1 for parametres $x_{5}^{\prime}, x_{6}^{\prime}, x_{7}^{\prime}$ and $x_{8}^{\prime}$ broken down into data correctly and incorrectly classified. The results indicate that in almost $90 \%$ cases of errors (precisely $86.89 \%$ ) people of the same gender were confused. In the case of correct classification, the result obtained is approximately equal to the expected value of $50 \%$. The achieved conclusion is confirmed in e.g. (Yu et al., 2009) where the differences in gait of different genders were indicated.

For parametres describing the identity of emotions, physical condition and past injuries of both the participant and the person representing the model in the training set, only slightly higher value of incorrect classification was noted $(25.8 \%$ for correct classification and $27.87 \%$ for incorrect classification in 'the same emotions', $50.69 \%$ and $55.74 \%$ respectively in the case of 'the same physical condition' and $51.87 \%$ and $59.32 \%$ for "past injuries").

Attention should, however, be paid to the fact that the results are processed based on the data collected from questionnaire filled out by the participants and, as a result, should be treated 
with some suspicion. Therefore, to be able to describe the influence of a particular parameter on the performance of the biometric system, the difference between two groups being analysed must be noticable enough so as not to cast any reservation. In the situation under analysis, it can be concluded that the biometric system has less problems with correct recognition when only one of the people being compared has had an injury in the past or has a dysfunction of the motor system influencing the movement pattern. This conclusion can be confirmed e.g. in Derlatka and Ihnatouski (2010) where the possobility for a quite accurate automatic recognition of a limited number of motor system dysfunctions was shown.

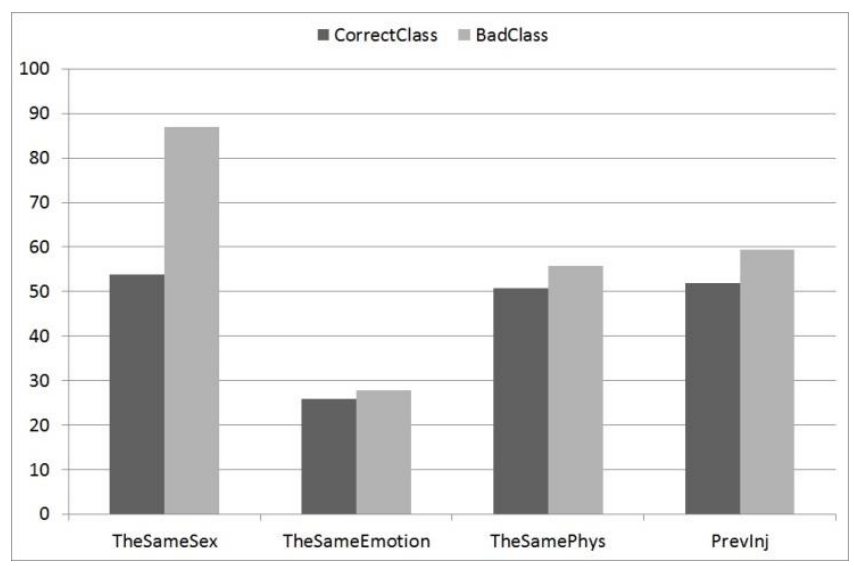

Fig. 1. The precentage contribution of parameters $x_{5}^{\prime}, x_{6}^{\prime}, x_{7}^{\prime}, x_{8}^{\prime}$ in both cases: correct recognition (CorrectClass) and bad recognition (BadClass)

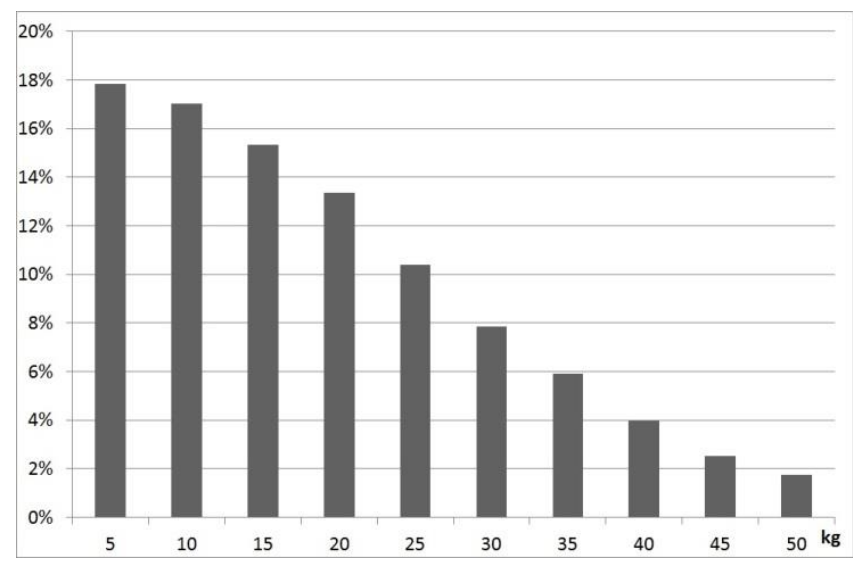

Fig. 2. Normalise histogram of the difference in the BW of relevant subjects in the case of correct recognition

The comparison of normalised histograms of the difference in the BW of relevant people in the case of both correct and incorrect classification clearly indicates that the incorrect classification in only possible when the difference is minor. In the analysed data, the maximum difference in the incorrect classification is $5.3 \mathrm{~kg}$. It it worth pointing out that in the case of correct classification the analysed data take a form similar to the normal distribution. This demonstrates that there is a close relationship between the difference in weight of individuals and the possibility for error occurence. It should be stressed out, that it is in line with the results presented in Jenkins and Ellis (2007) and intuition as the GRF values are proportional to the weight of the participant.

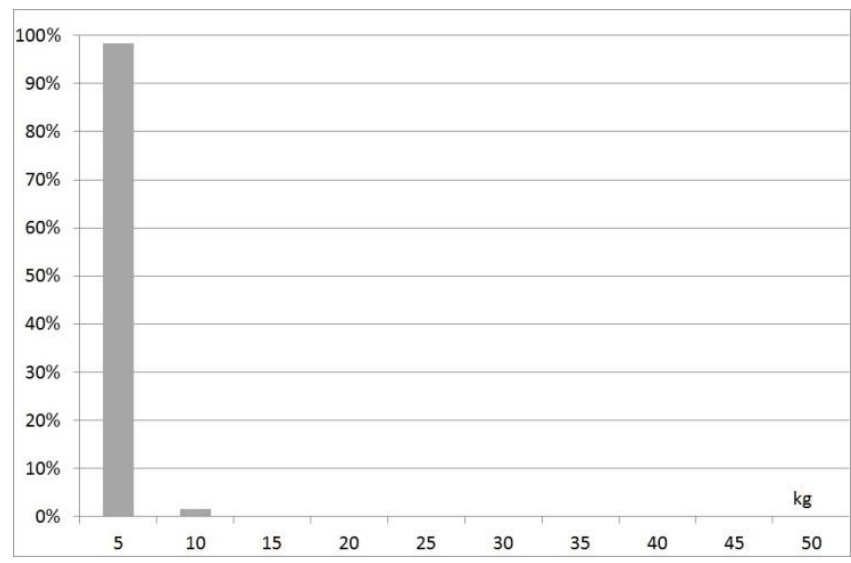

Fig. 3. Normalise histogram of the difference in the BW of relevant subjects in the case of bad recognition

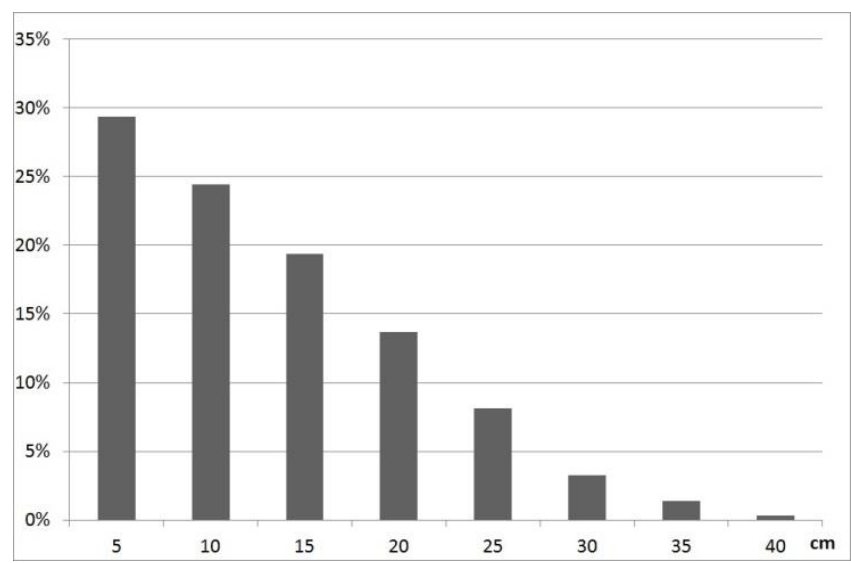

Fig. 4. Normalise histogram of the difference in the $\mathrm{BH}$ of relevant subjects in the case of correct recognition

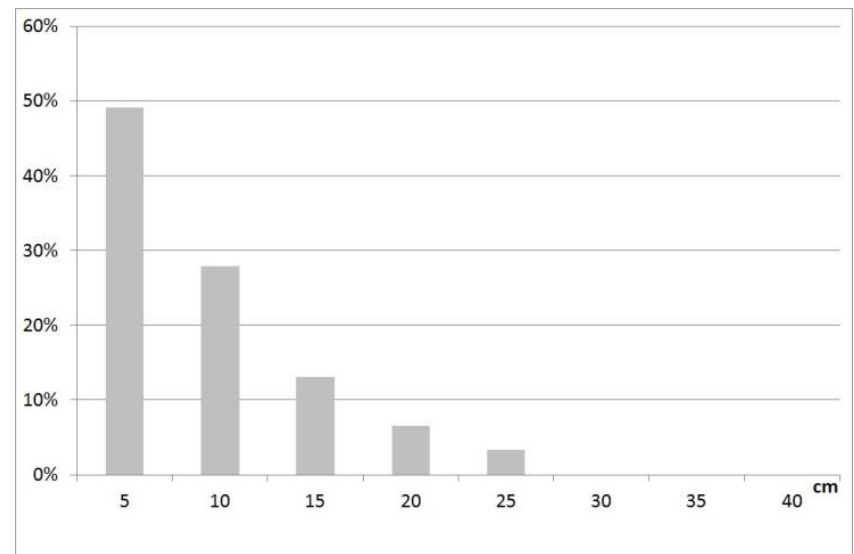

Fig. 5. Normalise histogram of the difference in the $\mathrm{BH}$ of relevant subjects in the case of bad recognition

The analysis of the charts shown in the figures 4 and 5 indicates, that the distribution of the data presented differs between the correct and incorrect classifications. This concerns not only the scope of the data (the maximum diffierence in $\mathrm{BH}$ in the incorrect classifications is $23.3 \mathrm{~cm}$ whereas in the case of correct classification this limit is exceeded in $8 \%$ of cases) but also the character of the distribution - the normal disctribution for the correctly classified data and the exponential distribution for errors. In this case the $U$ Mann-Whitney test was also carried out and it confirmed that this value is in a statistically significant way different in both groups. 


\section{CONCLUSIONS}

The study is an analysis of the influence of selected factors on the quality of recognition of the biometric system based on human gait. In order to enable the search for the connections between these parametres and the incorrect recognition, data describing the gait of almost 180 people was collected.

Summarising the results it should be concluded that there is no significant influence of parametres connected with participants' emotions and physical condition on the quality of the classification. It was also concluded that the biometric system has less problems with correct recognition when only one of the people being compared has had an injury in the past or has a dysfunction of the motor system influencing the movement pattern.

The most significant influence was observed in the case of the weight of the participants. During recognition, only people with a difference in weight of less than $5.3 \mathrm{~kg}$ were confused. A slightly less important influence was noticed in the case of both gender and body height of the participants. The author is, of course, aware of the fact that people of the same weight are most often of the same sex and of similar height. Irregardless, it is planned to conduct an analysis which will allow for the elimination of the influence of the difference in body weight on both of these factors. It was also noted, that people of the same gender and similar BH were confused most often.

The author is convincied that this knowledge allows for the design of more accurate biometric systems in the future.

\section{REFERENCES}

1. Balista J. A. F., Soriano M. N., Saloma C.A (2010), Compact timeindependent pattern representation of entire human gait cycle for tracking of gait irregularities, Pattern Recognition Letters, 31, 20-27.

2. Derlatka M. (2012), Human gait recognition based on signals from two force plates, ICAISC'2012 - LNCS:LNAI, 7268, 251-258.

3. Derlatka M. (2013), Modyfied kNN algorithm for improved recognition accuracy of biometrics system based on gait, CISIM'2013 - LNCS, 8104, 59-66.

4. Derlatka M., Ihnatouski M. (2010), Decision tree approach to rules extraction for human gait analysis, ICAISC'2010 - LNCS:LNAI, 597-604.

5. Gafurov D., Bours P., Snekkenes E. (2011) User authentication based on foot motion, Signal, Image and Video Processing, 5, 457-467.

6. Goldy F. R, Mary R. P. (2012), Genetic Algorithm for self occlusion gait recognition, International Journal of Advanced Research in Computer and Communictation Engineering, 1, 10, 865-869.

7. Idźkowski A., Walendziuk W. (2009), Evaluation of the static posturograph platform accuracy, Journal of Vibroengineering, 11, 3, 511-516.
8. Jenkins J., Ellis C. (2007), Using ground reaction forces from gait analysis: body mass as a weak biometrics, LNCS, 4480, 251-267.

9. Katiyar R., Pathak V. K., Arya K. V. (2013), A study on existing gait biometrics approaches and challenges, International Journal of Computer Science, 10(1), 135-144.

10. Klempous R. (2012), The different possibilities for gait identification based on motion capture, EUROCAST, LNCS, 6928, 187-194.

11. Kumar A., Ramakrishnan M. (2011), Legacy of footprints recognition - a review, International Journal of Computer Applications, 35(11), 9-16.

12. Lin Y. C, Lin Y. T. (2013), Human recognition based on plantar pressure patterns during gait, $\mathrm{J}$. of Mechanics in Medicine and Biology, 13(2).

13. Lin Y. C, Yang B. S, Lin Y. T, Yang Y. T. (2011) Human recognition based on kinematics and kinetics of gait, Journal of Medical and Biological Engineering, , 31(4), 255-263.

14. Moustakidis S. P, Theocharis J. B., Giakas G. (2009), Feature extraction based on a fuzzy complementary criterion for gait recognition using GRF signals, $17^{\text {th }}$ Mediterranean Conference on Control \& Automation, Greece, IEEE, 1456-1461.

15. Nakajima K., Mizukami Y., Tanaka K., Tamura T. (2000), Footprintbased personal recognition, IEEE Transactions on Biomedical Engineering, 47(11),1534-1537.

16. Pataky T. C, Mu T., Bosch K., Rosenbaum D., Goulermas J. Y. (2012), Gait recognition: highly unique dynamic plantar pressure patterns among 104 individuals, J. R. Soc. Interface, 9(69), 790-800.

17. Porwik P., Zygula J., Doroz R, Proksa R. (2010), Biometric recognition system based on the motion of the human body gravity centre analysis, Journal of Medical Informatics and Technologies, 15, 61-69.

18. Rodriguez V. R., Manson J., Evans N. (2009), Assessment of a footstep biometric verification System Handbook of Remote Biometrics, eds. Tistarelli et al. Advances in Pattern Recognition, London, 313-327.

19. Switonski A., Polanski A., Wojciechowski K.. (2011), Human identification based on gait paths, ACIVS LNCS, 6915, 531-542.

20. Wu J., Wang J., Liu L. (2007), Feature extraction via KPCA for classification of gait patterns, Human Movement Science, 26, $393-411$.

21. Xu X., Tang J., Zhang X., Liu X., ZXhang H., Qiu Y. (2013), Exploring techniques for vision based human activity recognition: methods, systems and evaluation, Sensors, 13, 1635-1650.

22. Yao Z. M., Zhou X., Lin E. D, Xu S., Sun Y. N. (2010), A novel biometric recognition system based on ground reaction force measurements of continuous gait. Human System Interactions, $3^{\text {rd }}$ Conf. on Digital Object Identifier, Rzeszow Poland, 452-458.

23. Yu S., Tan T., Huang K., Jia K., Wu X., (2009), A study on gaitbased gender classification, IEEE Transactions on Image Processing, 18, 8, 1905-1910.

24. Yun J. (2011), User Identification using gait patterns on UbiFloorll, Sensors, 11, 2611-2639. 